\title{
Meditation Practice Is Associated with a Values-Oriented Life: the Mediating Role of Decentering and Mindfulness
}

\author{
Alba Franquesa ${ }^{1,2}$ - Ausiàs Cebolla ${ }^{3}$ - Javier García-Campayo ${ }^{4}$ Marcelo Demarzo $^{5}$. \\ Matilde Elices $^{6,7}$ • Juan Carlos Pascual ${ }^{2,6}$ • Joaquim Soler ${ }^{6,7}$
}

(C) Springer Science+Business Media New York 2017

\begin{abstract}
Clarification of personal values and meditation practice has been associated in most meditation traditions and in academic texts. Both values-related behavior and meditation practice increases well-being, but their relationship has not been well studied. It has been suggested that values, together with self-regulation, psychological flexibility, and exposure, are key mechanisms of action in mindfulness. The aims of this study were to explore the association between meditation and values and to examine the processes involved in this relation. A sample of 235 meditation-naïve participants and 274 subjects with varying levels of experience in meditation practice completed an online assessment protocol
\end{abstract}

Joaquim Soler

jsolerri@santpau.cat

1 Les Corts Centre d'Higiene Mental, Barcelona, Spain

2 Departament de Psiquiatria i Medicina Legal, Universitat Autònoma de Barcelona, Barcelona, Spain

3 Department of Personality, Evaluation and Psychological Treatments, Universitat de Valencia, Valencia, Spain

4 Department of Psychiatry, Miguel Servet Hospital \& University of Zaragoza, Instituto Aragonés de Ciencias de la Salud, Red de Actividades Preventivas y de Promoción de la Salud (REDIAPP), Zaragoza, Spain

5 "Mente Aberta"-Brazilian Center for Mindfulness and Health Promotion, Universidade Federal de São Paulo (UNIFESP), São Paulo, Brazil

6 Servei de Psiquiatria, Hospital de la Santa Creu i Sant Pau, Universitat Autònoma de Barcelona (UAB), Centro de Investigación Biomédica en Red de Salud Mental (CIBERSAM) (Madrid), Av. Sant Antoni M ${ }^{\mathrm{a}}$ Claret 167, Barcelona 08025, Spain

7 Departament de Psicología Clínica y de la Salut, Universitat Autònoma de Barcelona, Barcelona, Spain composed of Five Facets of Mindfulness Questionnaire (FFMQ), Decentering Questionnaire (EQ), Valued Living Questionnaire (VLQ), and Engagement with Life Scale (ELS). Results revealed that daily meditators were more consistent, aware, and life-fulfilled about their values; moreover, these measures correlated with the mindfulness process and decentering. The relation between meditation practice and values-related behavior (assessed by the VLQ) was mediated by decentering. The association between meditation practice and Valued Living and Life Fulfillment (measured by the ELS) was mediated by the decentering, describing, and nonjudging dimensions of mindfulness. The findings in this study support the relation between meditation and personal values, mediated by the decentering, describing, and non-judging facets of mindfulness.

Keywords Meditation $\cdot$ Mindfulness $\cdot$ Values $\cdot$ Decentering

\section{Introduction}

The role of personal values in modern psychotherapy has become increasingly important in recent years. Schwartz and Bilsky (1987) defined values as follows: "Values (a) are concepts or beliefs, (b) pertain to desirable end states or behaviors, (c) transcend specific situations, (d) guide selection or evaluation of behavior and events, and (e) are ordered by relative importance."

Among contemporary models of psychotherapy, acceptance and commitment therapy (ACT) is probably the model that has most emphasized the relevance of applying values work to psychotherapy (Hayes et al. 1999). ACT considers valued-oriented behavior to be a primary therapeutic goal, and values are used to facilitate change (Hayes et al. 2001). 
Based on the ACT framework, other third-generation therapies (i.e., mindfulness and acceptance-based therapies) such as dialectical behavior therapy (DBT; Linehan 2014) and behavioral activation for depression (Jacobson et al. 2001) have also included values-oriented interventions. The notion of "wise mind" proposed in DBT (Linehan 1993, 2014) can be also considered an example of the link between mindfulness and values. Wise mind refers to the inner wisdom that we all have, and by practicing mindfulness, we are able to access to it in order to identify and use skillful means for attaining valued ends (Linehan 1993). Similar to the proposal from selfdetermination theory (Deci and Ryan 1985a, b; Sheldon and Krieger 2014) — which argues that an open awareness is necessary for behaviors that are consistent with one's needs, values, and interests - the first step in the process is to identify those values. Other approaches, such as the humanistic model (Maslow 1962; Rogers 1971) and motivational interviewing (Miller and Rollnick 1991), have also emphasized the importance of values in human psychology. For a more detailed discussion of the differences in the conceptualization of values among these various approaches, see Yadavaia and Hayes (2009) and Páez-Blarrina et al. (2006).

The beneficial effects of acting in accordance with personal values have been demonstrated in both clinical and nonclinical samples. In a sample of students, Brunstein (1993) show that commitment, attainability, and progress in goal achievement linked to personal values play important roles in subjective well-being. In fact, data from several other studies have confirmed that values-focused psychological interventions improve several aspects related to well-being, including pain tolerance (Branstetter-Rost et al. 2009), stress response (Crocker et al. 2008; Gregg et al. 2014), and quality of life and physical and emotional functioning (McCracken 2013; Michelson et al. 2011; Vowles et al. 2011).

Mindfulness can be defined as the self-regulation of attention to one's experiences in the present moment with curiosity, openness, and acceptance (Bishop et al. 2004). Mindfulness is also effective for several mental health conditions (Khoury et al. 2013) and in generating improvements in well-being (Carmody and Baer 2008; Shapiro et al. 2008). Although data are scant with regard to the relationship between values and mindfulness, some authors have identified an expected positive link: Meditation is believed to help in fostering a deeper awareness that may allow people to analyze goals to identify their deepest values and thus engage in wiser actions (Campos et al. 2016; Linehan 2014; Williams and Penman 2011).

The practice of meditation may facilitate recognition and clarification of values, and this has been suggested as a potential mechanism for the beneficial effects of mindfulness (Kocovski et al. 2009). Meditation could exert this beneficial effect by diminishing an individual's tendency to be on "automatic pilot" in which quick decisions influenced by external factors (e.g., pushes and pulls) are prioritized over internal values, thus narrowing consideration of the full range of options and responses; consequently, decisions and behaviors may not be directed towards an individual's true needs and values.

In one study involving mindfulness-based cognitive therapy for chronically depressed participants with a history of suicidality, many of the patients reported more clarity about their important goals, and this perceived clarity increased their confidence in their capacity to move in valued life directions (Crane et al. 2012). Shapiro et al. (2006) suggested that mindfulness practice produces certain shifts in the perception of internal experiences, usually referred to as the capacity for reperceiving or decentering. This capacity to separate oneself from one's thoughts allows the person to observe his or her experiences with greater clarity and therefore to choose more wisely in accordance with personal values (Kocovski et al. 2009; Shapiro et al. 2006). Decentering is believed to facilitate many health-related processes such as self-regulation, psychological flexibility, exposure, and values clarification. Supporting this notion, Carmody et al. (2009) showed that the association between mindfulness decentering and a reduction in physiological symptoms is mediated by values clarification and flexibility.

Few studies have investigated the relation between mindfulness, meditation, and values. Brown and Ryan (2003) found that individuals who scored higher on a mindfulness scale were more autonomous in their activity and less likely to be influenced by the demands and pressure of others. These individuals spend more time doing things that they truly value and enjoy. Brown and Kasser (2005) also showed that high dispositional mindfulness was related to more environmentally responsible behavior and that mindfulness and intrinsic values (together, but not separately) were relevant variables implicated in the covariance between self well-being and ecologically responsible behavior.

In this context, the aim of the present was to explore the relation between meditation practice and values-related behavior comparing subjects with meditative experience and without meditative experience and also explore decentering and mindfulness as mediational variables. We expected that meditation would have a positive effect on values-influenced conduct. We also expected that greater mindfulness practice would be positively associated with decentering and mindfulness capacities and that these capacities would, in turn, promote values-related behavior.

\section{Method}

\section{Participants}

We obtained data from 698 individuals. However, due to incomplete information about meditation practice, 189 of these were excluded from analysis. Thus, a total of 509 individuals 
completed the survey and were therefore included in the final study sample; of these, 235 did not practice meditation (nonmeditation practitioners (NMP)). In a previous study of our group, we found that the frequency of the practice was the parameter most related to measures of mindfulness and decentering (Soler et al. 2014a). Therefore, the subsample of meditators was divided considering how frequently they practiced: daily meditation practitioners (DMP; $n=143$ ), those that practice three to four times a week (3-4MP; $n=92)$, and those that practice $\leq 2$ times per week (2-LESSMP; $n=39$ ). Demographic data are displayed in Table 1.

Most participants practiced more than one type of meditation. The most common practices included body scan (14.2\%), vipassana (18.6\%), concentrative practices $(19.3 \%)$, informal practices $(27 \%)$, imagination practices $(38.3 \%)$, mantras (44.5\%), and compassion-based practices (49.6\%).

\section{Procedure}

Participants completed an assessment protocol via a commercial online survey system (www.surveymonkey.com). A link to this protocol was posted on several Spanish websites about mindfulness, meditation, and psychology (scientific associations, mindfulness associations, monasteries, etc.), as well as on non-professional social networks (i.e., Facebook).

The study protocol was approved by the hospital's Ethics Committee, and all participants signed a consent form indicating their willingness to participate. Participants were informed about the purpose of the study and told that their answers would be treated confidentially.

\section{Measures}

Sociodemographic and meditation practice information: Sociodemographic data, including age, sex, education, and meditation practice, were obtained. Frequency of meditation practice was assessed with a brief, purpose-designed questionnaire: Participants were asked to indicate the frequency of their meditation practice (daily, three to four times/week, $\leq 2$ times/week, or never).

The Valued Living Questionnaire (VLQ) (Wilson et al. 2011) is a two-part instrument to assess values-related behavior. First, participants rate the importance of 10 life domains (importance scores) on a 10-point Likert-style scale ( $1=$ not at all important; $10=$ extremely important), as follows: (1) family (other than parenting and intimate relations), (2) marriage/couples/intimate relations, (3) parenting, (4) friendship, (5) work, (6) education, (7) recreation, (8) spirituality, (9) citizenship, and (10) physical self-care. In the second part of the questionnaire, subjects rate the extent to which their behavior over the last week reflected their values $(1=$ not at all consistent; $10=$ extremely consistent; consistency scores). Responses from both of these domains (i.e., importance and consistency) were used to calculate a composite index (i.e., "Valued Living composite"), which helps to quantify the extent to which a participant is living congruently with his/ her values in everyday life. The Valued Living composite index was calculated by multiplying the importance and consistency responses for each domain and then calculating the mean of those scores. The resulting Valued Living composite scores range from 10 to 100 . In our sample, the internal consistency for this composite index was considered adequate $(\alpha=0.65)$.

The Engagement with Life Scale (ELS; Trompetter et al. 2013) is a 16-item scale, with each item rated on a five-point Likert scale ranging from 1 (completely disagree) to 5 (completely agree). Results provide a general underlying factor and two subscales: Valued Living (10 items) and Life Fulfillment (6 items). The scores on Valued Living reflect the recognition and knowledge of personal values and the underlying behavioral actions congruent with these values. The Life Fulfillment subscale is composed of items measuring the sense of fulfillment in life as a consequence of recognizing and living in accordance with personal values. In our sample, the

Table 1 Sociodemographic characteristics by group

\begin{tabular}{|c|c|c|c|c|c|}
\hline $\begin{array}{l}\text { Frequency of } \\
\text { meditation } \\
\text { practice }\end{array}$ & Daily $n=143$ & $\begin{array}{l}3 \text { or } 4 \text { times a } \\
\text { week } n=92\end{array}$ & $\begin{array}{l}\leq 2 \text { times a week } \\
n=39\end{array}$ & $\begin{array}{l}\text { No meditation } \\
\text { practice } n=235\end{array}$ & \\
\hline & Mean (SD) & Mean (SD) & Mean (SD) & Mean (SD) & $F(p)$ \\
\hline \multirow[t]{2}{*}{ Age } & $45.42(9.97)$ & $42.04(10.40)$ & $42.85(11.75)$ & $39.26(11.62)$ & $9.23(<0.0001)$ \\
\hline & $N(\%)$ & $N(\%)$ & $N(\%)$ & $N(\%)$ & $\chi^{2}(p)$ \\
\hline Sex & & & & & $2.47(0.48)$ \\
\hline Male & $51(35.7)$ & $29(31.5)$ & $13(33.3)$ & $66(28.1)$ & \\
\hline Female & $92(64.3)$ & $63(68.5)$ & $26(66.7)$ & $169(71.9)$ & \\
\hline Education & & & & & $8.24(0.22)$ \\
\hline Primary & $1(.7)$ & 0 & 0 & $4(1.7)$ & \\
\hline Secondary & $18(12.6)$ & $6(6.5)$ & $1(2.6)$ & $18(7.7)$ & \\
\hline University & $124(86.7)$ & $86(93.5)$ & $38(97.4)$ & $213(90.6)$ & \\
\hline
\end{tabular}


Cronbach's alpha for both subscales and the total scale of the ELS revealed excellent internal consistencies: 0.93 for the total scale, 0.90 for Valued Living, and 0.89 for Life Fulfillment.

The Five Facet Mindfulness Questionnaire (FFMQ; Baer et al. 2008) is an instrument based on five independently developed mindfulness questionnaires and consists of a 39-item scale rated on a five-point Likert scale $(1=$ never or very rarely true to $5=$ very often or always true) with five subscales: observe, describe, act with awareness, non-judge of inner experience, and non-react to inner experience. Observe refers to noticing or attending to internal and external experiences such as sensations, thoughts, or emotions. Describe refers to labeling internal experiences with words. Act with awareness describes focusing on activities in the moment as opposed to behaving mechanically. Non-judge of inner experience refers to taking a non-evaluative stance towards thoughts and feelings. Non-react to inner experience is defined as allowing thoughts and feelings to come and go, without getting caught up in or carried away by them. Cronbach's $\alpha$ for the Spanish version of the FFMQ range from 0.8 to 0.91 (Cebolla et al. 2012). In our sample, the Cronbach's $\alpha$ for the subscales ranged from 0.84 to 0.93 .

The Experiences Questionnaire-Decentering (EQ-D; Fresco et al. 2007) is an 11-item self-report measure of decentering. Items are rated on a five-point Likert scale $(1=$ never to $5=$ always). Spanish validation of the EQ-D scale showed high internal reliability (Cronbach's $\alpha=0.89$ ) (Soler et al. 2014b). In our sample, Cronbach's $\alpha$ was also 0.89 .

\section{Data Analyses}

To explore sociodemographic differences between groups, we used ANOVAs and chi-squared tests. Differences between groups in terms of VLQ, ELS, FFMQ, and EQ scores were examined by means of a multivariate ANOVA; in all cases, age was entered as a co-variable.

To examine the association between decentering and mindfulness (EQ and FFMQ) and values (VLQ and ELS), partial correlation analysis was carried out, controlling for the effect of age. Finally, to test if mindfulness mediates the relation between meditative practice and valued living, we conducted a hierarchical regression analysis for values-related tests (i.e., VLQ composite, Valued Living, and Life Fulfillment), including the following as predictors: age in the first step, meditation practice in the second step, and EQ-D and FFMQ subscales in the third step. Then, using Preacher and Hayes' (2004) approach for SPSS, multiple mediation analysis, adjusted for age, was applied to the values-related measures, including meditative practice as an independent variable, the valuesrelated measures as dependent variables, and the EQ-D and FFMQ subscales that were significant in the previous regression model as mediators. To test the hypothesis that EQ-D and
FFMQ subscales mediate the meditation-values relationship, a bootstrap regression analysis was carried out.

\section{Results}

\section{Differences in Sociodemographic Variables Between Groups with Different Meditation Frequency}

Analysis of sociodemographic variables revealed significant differences between groups for age, indicating that this variable should be controlled in the subsequent analyses. No differences were found for level of education or sex. Table 1 shows the sociodemographic characteristics of each group.

\section{Between-Group Differences in Values-Related Measures, Mindfulness, and Decentering}

Results of the MANCOVA show significant differences between groups on the VLQ composite, the Valued Living subscale, and the Life Fulfillment subscale. Post hoc analyses showed that daily meditators had significantly higher scores on all values-related measures. However, no significant differences between non-meditators and the other two groups (i.e., meditative practice twice a week or less and meditative practice three or four times a week) were observed. Effect sizes for significant differences in the VLQ composite, Valued Living, and Life Fulfillment between groups were small or medium (ranging from 0.40 to 0.60 ) (Table 2).

Significant between-group differences were also observed for EQ-D and all FFMQ scores. Effect sizes between daily meditators and non-meditators on the EQ-D and FFMQ scores were medium or large. Significant differences were also observed between the groups of meditators on several variables: EQ-D, FFMQ observe, FFMQ act with awareness, and FFMQ non-react to inner experience. (Table 2 shows post hoc analyses and effect sizes).

\section{Correlation Between Decentering, Mindfulness, and Values}

Correlational analyses (Table 3) showed significant associations between decentering (EQ-D), mindfulness (FFMQ), and values-related measures. Correlations between Valued Living, Life Fulfillment, and mindfulness measures were moderate, while associations between VLQ and mindfulness were small.

\section{Predictive Effect of Meditation Practice, Decentering, and Mindfulness Facets on Values-Related Behavior}

Given the association between age and meditation practice, we included age in the regression models. Since our primary interest was to study the effects of meditation practice on 
Table 2 Differences between groups on the mean scores of values-related measures, EQ-D, and FFMQ controlled by age

\begin{tabular}{|c|c|c|c|c|c|c|c|}
\hline & \multicolumn{4}{|l|}{ Mean (SD) } & \multirow[t]{2}{*}{ MANCOVA $F$} & \multirow{2}{*}{$\begin{array}{l}\text { Significant post hoc } \\
\text { differences }\end{array}$} & \multirow[t]{2}{*}{ Cohen's $d$} \\
\hline & NMP & 2-LESSMP & $3-4 \mathrm{MP}$ & DMP & & & \\
\hline \multirow[t]{3}{*}{ VLQ composite } & \multirow[t]{3}{*}{$59.72(13.65)$} & \multirow[t]{3}{*}{$60.25(14.52)$} & \multirow[t]{3}{*}{$61.217(13.966)$} & \multirow[t]{3}{*}{$67.048(12.462)$} & \multirow[t]{3}{*}{$6.62 * *$} & $\mathrm{DMP}>3-4 \mathrm{MP}$ & 0.44 \\
\hline & & & & & & DMP > 2-LESSMP & 0.50 \\
\hline & & & & & & DMP > NMP & 0.56 \\
\hline \multirow[t]{3}{*}{ Valued Living } & \multirow[t]{3}{*}{$40.058(5.918)$} & \multirow[t]{3}{*}{$40.743(5.838)$} & \multirow[t]{3}{*}{$41.141(5.244)$} & \multirow[t]{3}{*}{$53.088(4.696)$} & \multirow[t]{3}{*}{$8.421 * *$} & $\mathrm{DMP}>3-4 \mathrm{MP}$ & 0.39 \\
\hline & & & & & & DMP > 2-LESSMP & 0.44 \\
\hline & & & & & & $\mathrm{DMP}>\mathrm{NMP}$ & 0.57 \\
\hline \multirow[t]{2}{*}{ Life Fulfillment } & \multirow[t]{2}{*}{$21.447(4.604)$} & \multirow[t]{2}{*}{$22.514(3.752)$} & \multirow[t]{2}{*}{$22.411(4.338)$} & \multirow[t]{2}{*}{$24.056(4.045)$} & \multirow[t]{2}{*}{$9.044 * *$} & $\mathrm{DMP}>3-4 \mathrm{MP}$ & 0.39 \\
\hline & & & & & & $\mathrm{DMP}>\mathrm{NMP}$ & 0.60 \\
\hline \multirow[t]{5}{*}{ EQ-D } & \multirow[t]{5}{*}{$36.774(6.429)$} & \multirow[t]{5}{*}{$39.833(5.964)$} & \multirow[t]{5}{*}{$40.716(6.067)$} & \multirow[t]{5}{*}{$43.320(4.956)$} & \multirow[t]{5}{*}{$27.379 * *$} & $\mathrm{DMP}>3-4 \mathrm{MP}$ & 0.47 \\
\hline & & & & & & DMP > 2-LESSMP & 0.64 \\
\hline & & & & & & $\mathrm{DMP}>\mathrm{NMP}$ & 1.14 \\
\hline & & & & & & $3-4 \mathrm{MP}>\mathrm{NMP}$ & 0.63 \\
\hline & & & & & & 2-LESSMP > NMP & 0.49 \\
\hline \multirow[t]{5}{*}{ FFMQ observe } & $25.165(5.626)$ & $27.622(5.659)$ & $30.067(5.165)$ & $31.053(4.176)$ & $29.002 * *$ & DMP > 2-LESSMP & 0.69 \\
\hline & & & & & & DMP > NMP & 1.19 \\
\hline & & & & & & 3-4 > 2-LESSMP & 0.45 \\
\hline & & & & & & 3-4 > NMP & 0.91 \\
\hline & & & & & & 2-LESSMP > NMP & 0.43 \\
\hline FFMQ describe & $29.767(5.573)$ & $31.243(4.304)$ & $31.292(6.049)$ & $32.056(4.937)$ & $3.553 *$ & DMP > NMP & 0.44 \\
\hline & & & & & & $3-4 \mathrm{MP}>\mathrm{NMP}$ & 0.26 \\
\hline FFMQ act with & $27.470(5.960)$ & $26.027(5.833)$ & $27.595(5.738)$ & $29.962(5.092)$ & $5.578 * *$ & $\mathrm{DMP}>3-4 \mathrm{MP}$ & 0.4 \\
\hline awareness & & & & & & DMP > 2-LESSMP & 0.72 \\
\hline & & & & & & $\mathrm{DMP}>\mathrm{NMP}$ & 0.45 \\
\hline FFMQ non-judge & $28.262(6.692)$ & 31.918 (6.139) & $31.820(7.204)$ & $33.106(5.695)$ & $13.847 * *$ & $\mathrm{DMP}>\mathrm{NMP}$ & 0.78 \\
\hline & & & & & & $3-4 \mathrm{MP}>\mathrm{NMP}$ & 0.51 \\
\hline & & & & & & 2-LESSMP > NMP & 0.57 \\
\hline FFMQ non-react & $21.379(4.606)$ & $23.135(3.875)$ & $24.101(4.131)$ & $26.045(3.673)$ & $24.034 * *$ & $\mathrm{DMP}>3-4 \mathrm{MP}$ & 0.50 \\
\hline & & & & & & DMP > 2-LESSMP & 0.77 \\
\hline & & & & & & $\mathrm{DMP}>\mathrm{NMP}$ & 1.12 \\
\hline & & & & & & $3-4 \mathrm{MP}>\mathrm{NMP}$ & 0.62 \\
\hline & & & & & & 2-LESSMP > NMP & 0.41 \\
\hline
\end{tabular}

$N M P$ not meditative practice, 2-LESSMP meditative practice twice a week or less, 3-4MP meditative practice two or four times a week, $D M P$ daily meditative practice

$* p \leq 0.05 ; * p \leq 0.005$

values-related behaviors, the first model included only age and meditation practice. In a second step, we examined whether meditation practice, decentering, and mindfulness facets contribute independently to the prediction of values-

Table 3 Partial correlations, controlled by age

\begin{tabular}{llllllll}
\hline & EQ & $\begin{array}{l}\text { FFMQ } \\
\text { observe }\end{array}$ & $\begin{array}{l}\text { FFMQ } \\
\text { describe }\end{array}$ & $\begin{array}{l}\text { FFMQ act } \\
\text { aware }\end{array}$ & $\begin{array}{l}\text { FFMQ } \\
\text { non-judge }\end{array}$ & FFMQ non-react & $\begin{array}{l}\text { VLQ composite } \\
\text { Valued } \\
\text { Living }\end{array}$ \\
\hline VLQ composite & $0.305^{* *}$ & $0.191^{* *}$ & $0.188^{* *}$ & $0.107^{*}$ & $0.176^{* *}$ & $0.212^{* *}$ & - \\
Valued Living & $0.528^{* *}$ & $0.284^{* *}$ & $0.438^{* *}$ & $0.349 * *$ & $0.379 * *$ & $0.446^{* *}$ & $0.494 * *$ \\
Life Fulfillment & $0.552^{* *}$ & $0.348^{* *}$ & $0.371^{* *}$ & $0.335^{* *}$ & $0.391 * *$ & $0.434 * *$ & $0.475^{* *}$ \\
\hline
\end{tabular}

$* p \leq 0.05 ; * * p \leq 0.005$ 
related behavior (as assessed by the VLQ composite, Valued Living, and Life Fulfillment). Models included VLQ composite, Valued Living, and Life Fulfillment as dependent variables. In the first step, meditation practice was a significant predictor of all values-related behavioral measures: The models explained $4 \%$ of VLQ composite, $7 \%$ of Valued Living, and $8 \%$ of Life Fulfillment. In the second step, when EQ-D and FFMQ subscales were included as independent variables, meditation practice was no longer statistically significant in the three models. EQ-D was the only significant predictor of VLQ composite, with the model explaining $10 \%$ of the variance. EQ-D, FFMQ describe, and FFMQ non-judge were significant predictors of Valued Living and Life Fulfillment, explaining 37\% of the variance for Valued Living and $35 \%$ of Life Fulfillment.

\section{Mediational Role of Decentering and Mindfulness Between Meditation and Values}

In our proposed mediation models, we included meditation practice as the independent variable, while EQ-D and the significant FFMQ facets in the previous regression models (describe and non-judge) were included as mediation variables, with VLQ composite, Valued Living, and Life Fulfillment as dependent variables. Because of the significant relationship shown earlier between age and meditation practice, age was included as a co-variable.

According to Baron and Kenny (1986), several conditions are necessary to support a mediational hypothesis. First, if the data were collected at a single time point, there must be a theoretical basis to justify the role for each variable in the model. We tested the idea that meditation practice increases mindfulness skills and decentering, which, in turn, led to improved awareness of values and values-related behavior. In addition, the independent, mediator, and dependent variables must be significantly inter-correlated. This condition was confirmed in a previous correlational and regression analysis and in several previous publications (Baer et al. 2008; Soler et al. 2014a). Finally, when the independent variable and mediator variables are entered simultaneously in the model to predict the dependent variables (VLQ composite, Valued Living, and Life Fulfillment), the relationship between the independent variable and the dependent variables must be reduced. This condition was confirmed in the second step of the regression model (Table 4).

Mediational analysis (Fig. 1a-c) shows that meditation practice has an effect on VLQ composite, Valued Living, and Life Fulfillment; however, the direct effect of meditation practice on values was not significant when mediational variables were included in the analysis; otherwise, indirect effects remain significant. The indirect effect of meditative practice on decentering was shown to be significant on the VLQ composite, and other indirect effects of meditative practice (decentering, FFMQ describe, and FFMQ non-judge) remain significant on Valued Living and Life Fulfillment.

Bootstrapping analysis shows a significant indirect effect of meditation practice (by decentering) on the VLQ composite (95\% bootstrap confidence interval of $0.06-0.17$ ). For Valued Living, indirect effect of meditation practice was significant, as follows: decentering (95\% bootstrap confidence interval of $0.10-0.22$ ), describe (95\% bootstrap confidence interval of $0.02-0.08$ ), and non-judge (95\% bootstrap confidence interval of 0.01-0.08). For Life Fulfillment, the bootstrapping analysis also showed significant indirect effects of meditative practice as follows: decentering (95\% bootstrap confidence interval of $0.12-0.23$ ), describe ( $95 \%$ bootstrap confidence interval of $0.01-0.06$ ), and non-judge ( $95 \%$ bootstrap confidence interval of 0.02-0.69). In summary, bootstrap regression analysis supports the model of decentering as a partial mediator between meditation practice and VLQ composite scores and also for decentering, describe, and nonjudge as independent partial mediators on the effect of meditation practice on Valued Living and Life Fulfillment.

\section{Discussion}

As expected - and previously described in many meditation traditions - our findings indicate that there is a relation between meditation practice and values. Interestingly, compared to the less frequent meditators, the daily meditation group showed a greater capacity for behaving according to values and also higher scores on Life Fulfillment. Importantly, no significant differences on any of the values-related variables were found between subjects who practiced meditation less frequently and the mediation-naïve subjects. These results suggest that daily meditation is necessary to significantly influence values.

We also found that, in most cases, meditation practice had a significant impact on decentering and on all mindfulness indexes - even when practice was less than daily — particularly on decentering scores and on the observing and non-react facets of the FFMQ. These results are congruent with previous studies which have reported a relation between mindfulness practice and improvements on these facets (Soler et al. 2014a).

Decentering and all mindfulness facets (describe, non-react, act with awareness, non-judge, and observe) also correlated with values-related measures. Although meditative practice has an impact on values-related behavior (recognition, behavior congruence, and life fulfillment), when decentering and mindfulness facets are considered as a whole, they have the greatest influence on values-related measures; this is particularly true for decentering, describe, and non-judge, which are the three facets that determine a large part of values identification, committed behavior, and life fulfillment, regardless of the direct influence of meditative practice. 
Table 4 Three hierarchical regression analysis

\begin{tabular}{|c|c|c|c|c|c|c|c|c|c|}
\hline & $R^{2}$ & $\begin{array}{l}\text { Age } \\
\text { Beta }\end{array}$ & $\begin{array}{l}\text { Medit prac. } \\
\text { Beta }\end{array}$ & $\begin{array}{l}\text { EQ-D } \\
\text { Beta }\end{array}$ & $\begin{array}{l}\text { FFMQ obs. } \\
\text { Beta }\end{array}$ & $\begin{array}{l}\text { FFMQ desc. } \\
\text { Beta }\end{array}$ & $\begin{array}{l}\text { FFMQ act. } \\
\text { Beta }\end{array}$ & $\begin{array}{l}\text { FFMQ non-jud. } \\
\text { Beta }\end{array}$ & $\begin{array}{l}\text { FFMQ non-re. } \\
\text { Beta }\end{array}$ \\
\hline \multicolumn{10}{|l|}{ Step 2} \\
\hline VLQ & 0.05 & 0.07 & $0.21 * *$ & & & & & & \\
\hline Val. Liv. & $0.07 * *$ & $0.14 * *$ & $0.20 * *$ & & & & & & \\
\hline Life Ful. & $0.08 * *$ & $0.13 * *$ & $0.22 * *$ & & & & & & \\
\hline \multicolumn{10}{|l|}{ Step 3} \\
\hline VLQ & $0.10 * *$ & 0.05 & 0.09 & $0.28 * *$ & -0.01 & 0.07 & -0.05 & 0.05 & -0.05 \\
\hline Val. Liv. & $0.37 * *$ & $0.09 *$ & -0.03 & $0.34 * *$ & -0.06 & $0.22 * *$ & 0.07 & $0.12 *$ & 0.07 \\
\hline Life Ful. & $0.35^{* *}$ & 0.08 & -0.03 & $0.40 * *$ & 0.02 & $0.13^{* *}$ & 0.05 & $0.18^{* *}$ & -0.01 \\
\hline
\end{tabular}

Medit prac. meditative practice, FFMQ obs. FFMQ observe, FFMQ desc. FFMQ describe, FFMQ act. FFMQ act with awareness, FFMQ non-jud. FFMQ non-judge of inner experience, FFMQ non-re. FFMQ non-react to inner experience, VLQ VLQ composite, Val. Liv. Valued Living, Life Ful. Life Fulfillment

$* p \leq 0.05 ; * p \leq 0.005$

Interestingly, while meditative practice does not show a direct relation with values when mindfulness facets and decentering are included as independent variables, meditative practice improves all of these process variables. Mediational analysis supports this finding, since the direct effects of meditative practice on values disappear when mediational variables (decentering, describe, and non-judge) are considered. It is important to note that mediational variables are influenced by meditative practice in all cases and that the indirect effects of mediational variables from meditative practice are high. At the same time, mediational variables also have a direct impact, regardless of meditative practice, which leads us to believe that other therapies or practices that increase decentering, describing, and non-judging, such as meta-cognition-based therapies, CBT (Teasdale et al. 2002) or even dispositional mindfulness (Brown and Ryan 2003; Nitzan-Assayag et al. 2015), or education (Soler et al. 2014a) can influence values.

The theoretical model proposed by Shapiro et al. (2006) hypothesized that changes in reperceiving (decentering) mediate changes in values clarification, flexibility, self-regulation, and exposure, which, in turn, contribute to well-being. Our results confirm the relationship between meditation practice and values mediated not only by the decentering process but also by other processes involved in mindfulness, such as describe and non-judge.

Our findings were similar to those observed by Baer et al. (2008), who found that describe, non-judge, and non-react were significant mediators between meditation experience and wellbeing. Three mediational processes appear again, and two of these coincide: acceptance operationalized by non-judge and verbal labeling operationalized by describe. Both of these are well-established processes involved in emotion regulation and well-being (Creswell et al. 2007; Kashdan et al. 2006).

The ability of being non-judgmental might be associated with the pursue of one's real interest, regardless of cognitive assessments (in terms of what is "right or wrong"). The concept of "judging" is embedded in behaviors that are guided for "what it should be done" rather than "what I would like to do," called language traps in ACT (Dahl et al. 2009). Differentiating between both types of behaviors is a common and fundamental issue for interventions that aimed at value clarification (Harris 2011). Additionally, judging has an important effect on emotions. By judging one's primary emotion - usually a natural and evolution-tailored reaction to a given situation - switches to a secondary emotional response, frequently related to the individual's learning experiences, rather than to the current context (the one that elicited the emotional response). These secondary responses are less likely to be adaptive (Fruzzetti and Iverson 2006; Greenberg and Johonson 1990), for example, feeling anger for having felt rejection on a given social interaction or feeling shame for having felt fear. These changes in the emotional response entail changes in the expression, motivation, and aims, easily leading to a confusion on our inner interests (Fuzzeti and Iverson 2006; Greenberg and Johnson 1990). The capacity of non-judging and being able to skillfully describe the experience is crucial for a balanced self (Fruzzeti and Iverson 2006).

Describe refers to labeling the internal experience with words; this process tends to dampen affective responses (Lieberman et al. 2011; Linehan 1993) and would facilitate a more flexible response to address the intrinsic motivations of the individual. Description has the power to defuse the interference of judgments, secondary emotions, and misunderstandings (Fruzzetti and Iverson 2006; Linehan 2014).

The third element in Baer's study (2008) was non-react, decentering in our study, but both of these facets highly overlap (Soler et al. 2014b). Decentering allows the person to observe his or her experiences with greater clarity and therefore to choose wisely in accordance with personal values. The 

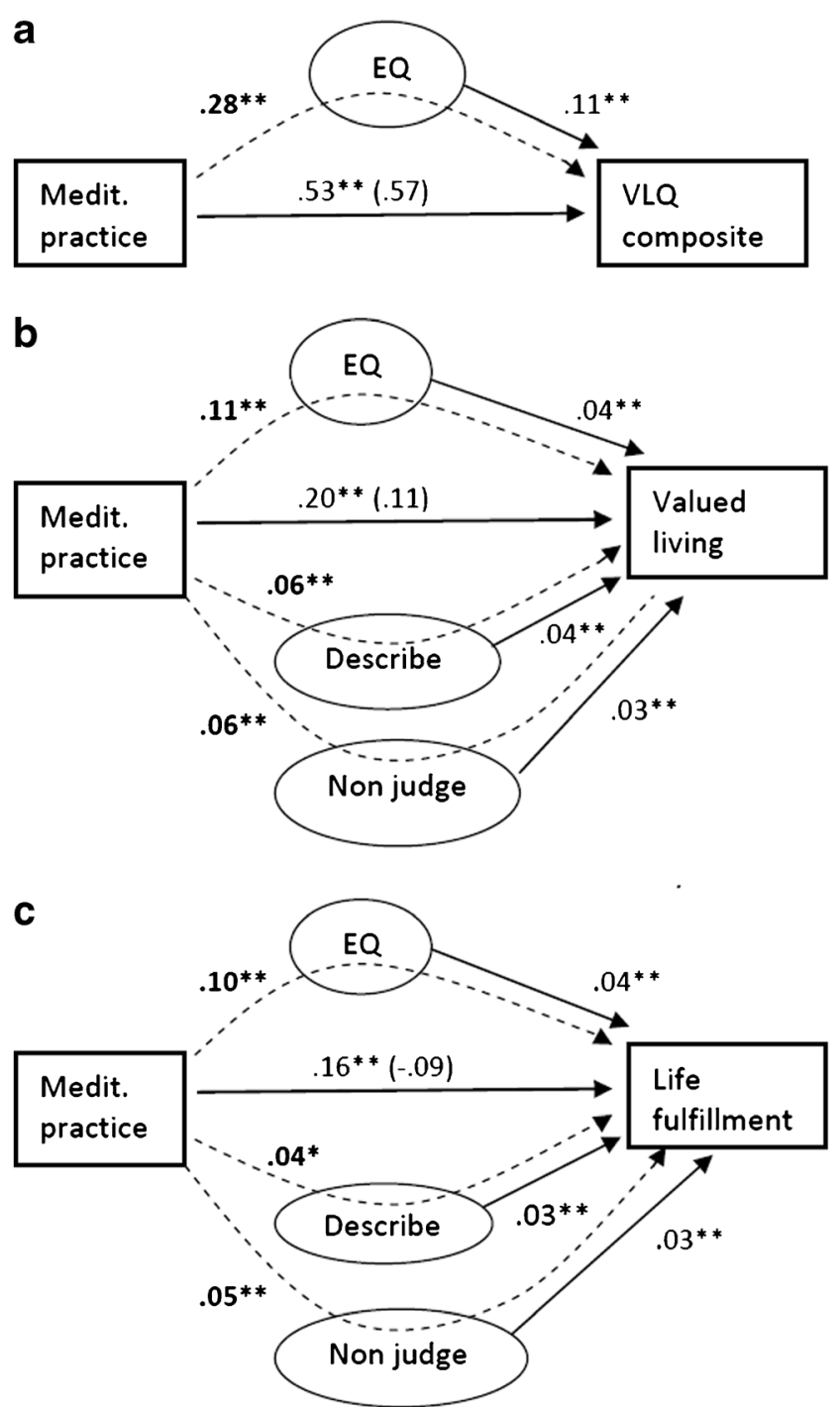

Fig. 1 a, b, c Three mediational analyses. All values are betastandardized coefficients. The values in parentheses show the relationship between meditative practice and values when indirect effect of $E Q$, FFMQ describe, and FFMQ non-judge are excluded. Signification of indirect effects was calculated by the Sobel test. ${ }^{*} p \leq 0.05$; $* * p \leq 0.005$. Medit. practice meditative practice

main limitation of this study is the design: Strictly speaking, mediation/moderation studies require at least two consecutive measures, which our study did not have. Mediation analysis with only one measure should be considered tentative and analyzed with caution (Judd et al. 2001). In addition, in our analysis, we did not consider the type of meditative practice and the duration of each practice session, both of which could have influenced our findings. Also, this is a cross-sectional study and characteristics of the sample, including the ability of maintaining a daily meditation routine, personality characteristics, or types and context of practice, could also be affecting these results. Although most participants were recruited in lay meditation centers, we do not know if the daily meditator group included individuals with religious formal training as for example monks. This might have impacted our study, and therefore, we consider this lack of information as a limitation. Moreover, considering the fundamentally experiential and temporal nature of values, the use of self-report questionnaires could easily be influenced by the desirability or preconceived ideas about oneself, especially in individuals with less awareness of the experience. In this sense, it would be desirable in future studies to find alternative methods of assessing values, such as implicit behavioral assessments, that may be more sensitive to experience and less influenced by judgments or social expectations. Finally, although some studies suggest that there are gender-related differences in values on some life domains (Bookwala et al. 2001), we did not perform a separate analysis based on gender.

Values have a high priority in mindfulness and acceptancebased psychotherapy (Germer et al. 2013; Roemer and Orsillo 2009). For some people, recognizing values is not easy, and therefore, at least part of therapeutic work needs to focus on identifying personal values. ACT and DBT, for example, both include exercises to help patients discover their values and to identify obstacles to achieving their values-related goals (Linehan 2014; Roemer and Orsillo 2009). In ACT, values and committed action are two out of the six core process that serve to increase psychological flexibility. All handbooks and protocols include techniques to clarify values and increase behavior regulation by it (Hayes 1999; Páez 2006). With regard to DBT, working with values is especially relevant for the fourth stage of individual therapy in which the work is focused in attaining a sense of connectedness, joy, and freedom (Linehan 1993). Clarifying the client's values is a core aspect to achieve this. In fact, to this end, the last version of DBTs' skills training includes several skills to help clients to work towards valued actions (Linehan 2014). The present study demonstrates that the association between meditative practice and values is primarily a question of improving mediational variables; as a result, any other practices or interventions that increase these capacities may also positively influence values. Other studies will be necessary to clarify the association between mediational variables and values and also the interaction between mediational variables and interventions specifically designed to clarify values and regulate behavior.

Acknowledgements We would like to thank all participants of the study. JS, AC, and JGC were supported by PROMOSAM: Investigación en procesos, mecanismos y tratamientos psicológicos para la promoción de la salud mental (Red de Excelencia PSI2014-56303REDT) founded by Ministerio de Economía y Competitividad (2014). The authors thank Bradley Londres for his assistance in editing and improving the text.

Author Contributions AF: designed the study, analyzed the data, and wrote the first draft of the paper. AC: collaborated in the design of the study, the data collection, and the data analyses. JGC: collaborated in the design of the study, the data collection, and the data analyses. MD: collaborated in the design of the study, the data collection, and writing of the 
final manuscript. ME: collaborated in the writing of the manuscript. JCP: collaborated in the writing of the final manuscript. JS: collaborated in the design, data analyses, and the writing of the final manuscript.

\section{Compliance with Ethical Standards}

Ethical Approval All procedures performed in studies involving human participants were in accordance with the ethical standards of the institutional and/or national research committee and with the 1964 Helsinki Declaration and its later amendments or comparable ethical standards.

Informed Consent Informed consent was obtained from all individual participants included in this study.

Conflict of Interest The authors declare that they have no conflict of interest.

\section{References}

Baer, R. A., Smith, G. T., Lykins, E., Button, D., Krietemeyer, J., Sauer, S., et al. (2008). Construct validity of the five facet mindfulness questionnaire in meditating and nonmeditating samples. Assessment, 15(3), 329-342.

Baron, R. M., \& Kenny, D. A. (1986). The moderator-mediator variable distinction in social psychological research: conceptual, strategic, and statistical considerations. Journal of Personality and Social Psychology, 51(6), 1173.

Bishop, S. R., Lau, M., Shapiro, S., Carlson, L., Anderson, N. D., Carmody, J., et al. (2004). Mindfulness: a proposed operational definition. Clinical Psychology: Science and Practice, 11, 230-241.

Bookwala, J., Coppola, K. M., Fagerlin, A., Ditto, P. H., Danks, J. H., \& Smucker, W. D. (2001). Gender differences in older adults' preferences for life-sustaining medical treatments and end-of-life values. Death Studies, 25(2), 127-149.

Branstetter-Rost, A., Cushing, C., \& Douleh, T. (2009). Personal values and pain tolerance: does a values intervention add to acceptance? The Journal of Pain, 10(8), 887-892.

Brown, K. W., \& Kasser, T. (2005). Are psychological and ecological well-being compatible? The role of values, mindfulness, and lifestyle. Social Indicators Research, 74(2), 349-368.

Brown, K. W., \& Ryan, R. M. (2003). The benefits of being present: mindfulness and its role in psychological well-being. Journal of Personality and Social Psychology, 84(4), 822.

Brunstein, J. C. (1993). Personal goals and subjective well-being: a longitudinal study. Journal of Personality and Social Psychology, 65(5), 1061.

Campos, D., Cebolla, A., Quero, S., Bretón-López, J., Botella, C., Soler, J., et al. (2016). Meditation and happiness: mindfulness and selfcompassion may mediate the meditation-happiness relationship. Personality and Individual Differences, 93, 80-85.

Carmody, J., \& Baer, R. A. (2008). Relationships between mindfulness practice and levels of mindfulness, medical and psychological symptoms and well-being in a mindfulness-based stress reduction program. Journal of Behavioral Medicine, 31(1), 23-33.

Carmody, J., Baer, R. A., Lykins, L. B. E., \& Olendzki, N. (2009). An empirical study of the mechanisms of mindfulness in a mindfulness based stress reduction program. Journal of Clinical Psychology, 65(6), 613-626.

Cebolla, A., Garcia-Palacios, A., Soler, J., Guillen, V., Baños, R., \& Botella, C. (2012). Psychometric properties of the Spanish validation of the Five Facets of Mindfulness Questionnaire (FFMQ). The European Journal of Psychiatry, 26(2), 118-126.

Crane, C., Winder, R., Hargus, E., Amarasinghe, M., \& Barnhofer, T. (2012). Effects of mindfulness-based cognitive therapy on specificity of life goals. Cognitive Therapy and Research, 36(3), 182-189.

Creswell, J. D., Way, B. M., Eisenberger, N. I., \& Lieberman, M. D. (2007). Neural correlates of dispositional mindfulness during affect labeling. Psychosomatic Medicine, 69(6), 560-565.

Crocker, J., Niiya, Y., \& Mischkowski, D. (2008). Why does writing about important values reduce defensiveness? Self-affirmation and the role of positive other-directed feelings. Psychological Science, 19(7), 740-747.

Dahl, J. C., Plumb, J. C., Stewart, I., \& Lundren, T. (2009). The art \& science of valuing in psychotherapy. Oakland: New Harbinger Publications.

Deci, E. L., \& Ryan, R. M. (1985a). The general causality orientations scale: self-determination in personality. Journal of Research in Personality, 19(2), 109-134.

Deci, E. L., \& Ryan, R. M. (1985b). Intrinsic motivation and selfdetermination in human behavior. New York: Plenum.

Fresco, D. M., Moore, M. T., van Dulmen, M. H., Segal, Z. V., Ma, S. H., Teasdale, J. D., \& Williams, J. M. G. (2007). Initial psychometric properties of the experiences questionnaire: validation of a selfreport measure of decentering. Behavior Therapy, 38(3), 234-246.

Fruzzetti, A. E., \& Iverson, K. M. (2006). Intervening with couples and families to treat emotion dysregulation and psychopathology. In D. K. Snyder, J. Simpson, \& J. N. Hughes (Eds.), Emotion regulation in couples and families: Pathways to dysfunction and health (pp. 249267). Washington, DC, US: American Psychological Association.

Germer, C. K., Siegel, R. D., \& Fulton, P. R. (Eds.). (2013). Mindfulness and psychotherapy. New York: Guilford Press.

Greenberg, L. S., \& Johnson, S. M. (1990). Emotional change processes in couples therapy. In E. A. Blechman (Ed.), Emotions and the family: For better or for worse (pp. 137-153). New Jersey: Routledge.

Gregg, J. A., Namekata, M. S., Louie, W. A., \& Chancellor-Freeland, C. (2014). Impact of values clarification on cortisol reactivity to an acute stressor. Journal of Contextual Behavioral Science, 3(4), 299-304.

Harris, R. (2011). The happiness trap. Auckland: Read HowYouWant.

Hayes, S. C., Strosahl, K. D., \& Wilson, K. G. (1999). Acceptance and commitment therapy. New York: Guilford Press.

Hayes, S. C., Barnes-Holmes, D., and Roche, B. (2001). Relational frame theory: a post-Skinnerian account of human language and cognition. Springer Science and Business Media.

Jacobson, N. S., Martell, C. R., \& Dimidjian, S. (2001). Behavioral activation treatment for depression: returning to contextual roots. Clinical Psychology: Science and Practice, 8(3), 255-270.

Judd, C. M., Kenny, D. A., \& McClelland, G. H. (2001). Estimating and testing mediation and moderation in within-subjects designs. Psychological Methods, 6, 115-134.

Kashdan, T. B., Barrios, V., Forsyth, J. P., \& Steger, M. F. (2006). Experiential avoidance as a generalized psychological vulnerability: comparisons with coping and emotion regulation strategies. Behaviour Research and Therapy, 44(9), 1301-1320.

Khoury, B., Lecomte, T., Fortin, G., Masse, M., Therien, P., Bouchard, V., et al. (2013). Mindfulness-based therapy: a comprehensive metaanalysis. Clinical Psychology Review, 33(6), 763-771.

Kocovski, N. L., Segal, Z. V., and Battista, S. R. (2009). Mindfulness and psychopathology: problem formulation. In Clinical handbook of mindfulness (pp. 85-98). New York: Springer.

Lieberman, M. D., Inagaki, T. K., Tabibnia, G., \& Crockett, M. J. (2011). Subjective responses to emotional stimuli during labeling, reappraisal, and distraction. Emotion, 11(3), 468.

Linehan, M. (1993). Cognitive-behavioral treatment of borderline personality disorder. New York: Guilford Press. 
Linehan, M. M. (2014). DBT skills training manual. New York: Guilford Publications.

Maslow, A. (1962). Toward a psychology of being. Princeton, NJ: D. Van Nostrana Co.

McCracken, L. M. (2013). Committed action: an application of the psychological flexibility model to activity patterns in chronic pain. The Journal of Pain, 14(8), 828-835.

Michelson, S. E., Lee, J. K., Orsillo, S. M., \& Roemer, L. (2011). The role of values consistent behavior in generalized anxiety disorder. Depression and Anxiety, 28(5), 358-366.

Miller, E. R., \& Rollnick, S. (1991). Motivational interviewing: preparing people to change addictive behavior. New York: Guildford Press.

Nitzan-Assayag, Y., Aderka, I. M., \& Bernstein, A. (2015). Dispositional mindfulness in trauma recovery: prospective relations and mediating mechanisms. Journal of Anxiety Disorders, 36, 25-32.

Páez-Blarrina, M., Gutiérrez-Martínez, O., Valdivia-Salas, S., \& LucianoSoriano, L. (2006). Terapia de Aceptación y Compromiso (ACT) y la importancia de los valores personales en el contexto de la terapia psicológica. Revista Internacional de Psicología y Terapia Psicológica, 6(1), 1-20.

Preacher, K. J., \& Hayes, A. F. (2004). SPSS and SAS procedures for estimating indirect effects in simple mediation models. Behavior Research Methods, Instruments, \& Computers, 36 (4), 717-731.

Roemer, L., \& Orsillo, S. M. (2009). Mindfulness-and acceptance-based behavioral therapies in practice. New York: Guilford Press.

Rogers, C. (1971). El proceso de convertirse en persona. Buenos Aires: Paidós.

Schwartz, S. H., \& Bilsky, W. (1987). Toward a theory of the universal content and structure of values: extensions and cross-cultural replications. Journal of Personality and Social Psychology, 58(5), 878.

Shapiro, S. L., Carlson, L. E., Astin, J. A., \& Freedman, B. (2006). Mechanisms of mindfulness. Journal of Clinical Psychology, 62(3), 373-386.

Shapiro, S. L., Oman, D., Thoresen, C. E., Plante, T. G., \& Flinders, T. (2008). Cultivating mindfulness: effects on wellbeing. Journal of Clinical Psychology, 64(7), 840-862.
Sheldon, K. M., \& Krieger, L. S. (2014). Walking the talk: value importance, value enactment, and wellbeing. Motivation and Emotion, 38(5), 609-619.

Soler, J., Cebolla, A., Feliu-Soler, A., Demarzo, M. M., Pascual, J. C., Baños, R., and García-Campayo, J. (2014a). Relationship between meditative practice and self-reported mindfulness: the MINDSENS composite index. PloS one, 9(1).

Soler, J., Franquesa, A., Feliu-Soler, A., Cebolla, A., García-Campayo, J., Tejedor, R., et al. (2014b). Assessing decentering: validation, psychometric properties, and clinical usefulness of the experiences questionnaire in a Spanish sample. Behavior Therapy, 45(6), 863871.

Teasdale, J. D., Moore, R. G., Hayhurst, H., Pope, M., Williams, S., \& Segal, Z. V. (2002). Metacognitive awareness and prevention of relapse in depression: empirical evidence. Journal of Consulting and Clinical Psychology, 70(2), 275.

Trompetter, H. R., Ten Klooster, P. M., Schreurs, K. M., Fledderus, M., Westerhof, G. J., \& Bohlmeijer, E. T. (2013). Measuring values and committed action with the Engaged Living Scale (ELS): psychometric evaluation in a nonclinical sample and a chronic pain sample. Psychological Assessment, 25(4), 1235.

Vowles, K. E., McCracken, L. M., \& O’Brien, J. Z. (2011). Acceptance and values-based action in chronic pain: a three-year follow-up analysis of treatment effectiveness and process. Behaviour Research and Therapy, 49(11), 748-755.

Williams, M., \& Penman, D. (2011). Mindfulness: a practical guide to finding peace in a frantic world. London: Hachette.

Wilson, K. G., Sandoz, E. K., Kitchens, J., \& Roberts, M. (2011). The Valued Living Questionnaire: defining and measuring valued action within a behavioral framework. The Psychological Record, $60(2), 4$.

Yadavaia, J. E., \& Hayes, C. (2009). Commitment therapy: a comparison with four other approaches. Hellenic Journal of Psychology, 6, 244 272. 